\title{
Deficiency of protein tyrosine phosphatase non-receptor type 2 in intestinal epithelial cells has no appreciable impact on dextran sulphate sodium colitis severity but promotes wound healing
}

Kasper, Stephanie H ; Spalinger, Marianne R ; Leonardi, Irina ; Gerstgrasser, Alexandra ; Raselli, Tina ; Gottier, Claudia ; Atrott, Kirstin ; Frey-Wagner, Isabelle ; Fischbeck-Terhalle, Anne ; Rogler, Gerhard ; Scharl, Michael

\begin{abstract}
BACKGROUND/AIMS The protein tyrosine phosphatase non-receptor type 2 (PTPN2) is known to mediate susceptibility to inflammatory bowel diseases. Cell culture experiments suggest that PTPN2 influences barrier function, autophagy and secretion of pro-inflammatory cytokines. PTPN2 knockout mice die a few weeks after birth due to systemic inflammation, emphasizing the importance of this phosphatase in inflammatory processes. The aim of this study was to investigate the role of PTPN2 in colon epithelial cells by performing dextran sulphate sodium (DSS)-induced colitis in PTPN2xVilCre mice. METHODS Acute colitis was induced by administering 2.5 or $2 \%$ DSS for 7 days and chronic colitis by 4 cycles of treatment using 1\% DSS. Body weight of mice was measured regularly and colonoscopy was done at the end of the experiments. Mice were sacrificed afterwards and colon specimens were obtained for Hamp;E staining. For analysis of wound healing, mechanical wounds were introduced during endoscopy and wound closure assessed by daily colonoscopy. RESULTS Although colonoscopy and weight development suggested changes in colitis severity, the lack of any influence of PTPN2 deficiency on histological scoring for inflammation severity after acute or chronic DSS colitis indicates that colitis severity is not influenced by epithelial-specific loss of PTPN2. Chronic colitis induced the development of aberrant crypt foci more frequently in PTPN2xVilCre mice compared to their wild type littermates. On the other hand, loss of PTPN2-induced enhanced epithelial cell proliferation and promoted wound closure. CONCLUSIONS Loss of PTPN2 in intestinal epithelial cells (IECs) has no significant influence on inflammation in DSS colitis. Obviously, loss of PTPN2 in IECs can be compensated in vivo, thereby suppressing a phenotype. This lack of a colitis-phenotype might be due to enhanced epithelial cell proliferation and subsequent increased wound-healing capacity of the epithelial layer.
\end{abstract}

DOI: https://doi.org/10.1159/000445289

Posted at the Zurich Open Repository and Archive, University of Zurich

ZORA URL: https://doi.org/10.5167/uzh-134538

Journal Article

Published Version

Originally published at:

Kasper, Stephanie H; Spalinger, Marianne R; Leonardi, Irina; Gerstgrasser, Alexandra; Raselli, Tina; Gottier, Claudia; Atrott, Kirstin; Frey-Wagner, Isabelle; Fischbeck-Terhalle, Anne; Rogler, Gerhard; Scharl, Michael (2016). Deficiency of protein tyrosine phosphatase non-receptor type 2 in intestinal 
epithelial cells has no appreciable impact on dextran sulphate sodium colitis severity but promotes wound healing. Digestion, 93(4):249-259.

DOI: https://doi.org/10.1159/000445289 


\title{
Deficiency of Protein Tyrosine Phosphatase Non-Receptor Type 2 in Intestinal Epithelial Cells Has No Appreciable Impact on Dextran Sulphate Sodium Colitis Severity But Promotes Wound Healing
}

\author{
Stephanie H. Kasper ${ }^{a} \quad$ Marianne R. Spalinger ${ }^{a} \quad$ Irina Leonardi ${ }^{a}$ \\ Alexandra Gerstgrasser ${ }^{a}$ Tina Raselli ${ }^{a}$ Claudia Gottier ${ }^{a}$ Kirstin Atrott ${ }^{a}$ \\ Isabelle Frey-Wagner ${ }^{\mathrm{a}} \quad$ Anne Fischbeck-Terhalle ${ }^{\mathrm{a}} \quad$ Gerhard Rogler $^{\mathrm{a}}$ \\ Michael Scharla, b \\ ${ }^{a}$ Division of Gastroenterology and Hepatology, University Hospital Zurich, ${ }^{b}$ Zurich Center for Integrative Human \\ Physiology, University of Zurich, Zurich, Switzerland
}

\section{Key Words}

Inflammatory bowel disease - Phosphatase non-receptor type 2 - Dextran sulphate sodium colitis · Proliferation

\begin{abstract}
Background/Aims: The protein tyrosine phosphatase nonreceptor type 2 (PTPN2) is known to mediate susceptibility to inflammatory bowel diseases. Cell culture experiments suggest that PTPN2 influences barrier function, autophagy and secretion of pro-inflammatory cytokines. PTPN2 knockout mice die a few weeks after birth due to systemic inflammation, emphasizing the importance of this phosphatase in inflammatory processes. The aim of this study was to investigate the role of PTPN2 in colon epithelial cells by performing dextran sulphate sodium (DSS)-induced colitis in PTPN2xVilCre mice. Methods: Acute colitis was induced by administering 2.5 or $2 \%$ DSS for 7 days and chronic colitis by 4 cycles of treatment using $1 \%$ DSS. Body weight of mice was measured regularly and colonoscopy was done at the end of the experiments. Mice were sacrificed afterwards and colon
\end{abstract}

specimens were obtained for H\&E staining. For analysis of wound healing, mechanical wounds were introduced during endoscopy and wound closure assessed by daily colonoscopy. Results: Although colonoscopy and weight development suggested changes in colitis severity, the lack of any influence of PTPN2 deficiency on histological scoring for inflammation severity after acute or chronic DSS colitis indicates that colitis severity is not influenced by epithelial-specific loss of PTPN2. Chronic colitis induced the development of aberrant crypt foci more frequently in PTPN2xVilCre mice compared to their wild type littermates. On the other hand, loss of PTPN2-induced enhanced epithelial cell proliferation and promoted wound closure. Conclusions: Loss of PTPN2 in intestinal epithelial cells (IECs) has no significant influence on inflammation in DSS colitis. Obviously, loss of PTPN2 in IECs can be compensated in vivo, thereby suppressing a phenotype. This lack of a colitis-phenotype might be due to enhanced epithelial cell proliferation and subsequent increased wound-healing capacity of the epithelial layer.

(c) 2016 S. Karger AG, Basel

\section{KARGER}

E-Mail karger@karger.com www.karger.com/dig (c) 2016 S. Karger AG, Base

0012-2823/16/0934-0249\$39.50/0
PD Dr. med. Michael Scharl

Division of Gastroenterology and Hepatology University Hospital Zurich

Rämistrasse 100, CH-8091 Zurich (Switzerland)

E-Mail michael.scharl@usz.ch 


\section{Introduction}

Crohn's disease (CD) and ulcerative colitis (UC) are the most common types of inflammatory bowel diseases (IBD), a group of chronic and relapsing inflammatory conditions of the intestine. Even though the multifactorial pathogenesis is still not entirely understood, it is generally accepted that IBD results from an excessive inflammatory immune response to otherwise commensal microbiota in genetically predisposed individuals. Genome-wide association studies helped to identify over 160 susceptibility loci for IBD [1]. Among these loci is the gene encoding protein tyrosine phosphatase non-receptor type 2 (PTPN2) that has also been reported to be linked to other autoimmune diseases such as diabetes type 1 and rheumatoid arthritis [2-4].

Similar to other phosphatases, PTPN2 is a negative regulator of many signalling pathways. It interferes with cytokine signalling by dephosphorylating some JAK and STAT molecules [5-7] and participates in growth regulation and glucose homeostasis by targeting EGFR and insulin receptor [5]. PTPN2 is a ubiquitously expressed protein that can be found in most mammalian embryonic and adult tissues with higher levels observed in lymphoid cells. In accordance with its potential role as IBD susceptibility gene, PTPN2 expression was found to be increased in intestinal biopsies of CD patients and to a lower extent also in UC patients [8]. Global loss of PTPN2 is lethal: $\mathrm{PTPN}^{-/-}$mice show defects in haematopoiesis, anaemia, massive tissue infiltration by mononuclear cells and die a few weeks after birth $[9,10]$. The embryonic development of these mice seems normal, the newborns are of normal size and health. Nevertheless, at the age of 3 weeks, they are obviously smaller compared to their wild type (wt) littermates and exhibit hunching, closed eyes, decreased mobility and diarrhoea followed by death [9].

From cell culture experiments using intestinal epithelial cell (IEC) lines it is already known that PTPN2 expression is increased by the pro-inflammatory cytokines TNF and IFN $\gamma[8,11,12]$. Knock down of PTPN2 potentiates the stimulatory effects of TNF and IFN $\gamma$ leading to exaggerated epithelial permeability and amplified secretion of interleukin (IL)-6 and IL-8 $[8,11,12]$. PTPN2 also regulates autophagy: loss of PTPN2 leads to impaired autophagosome formation and dysfunctional autophagy in IECs [13]. Without functional autophagy IECs are more prone to infection by intracellular invaders like Listeria monocytogenes [13].

The above-mentioned in vitro findings show the importance of PTPN2 in IECs and the knockout mouse demonstrates that PTPN2 also plays a role in inflammatory processes in vivo. Therefore, we investigated the in vivo role of PTPN2 in the intestinal epithelium. To do so, we generated a mouse strain with a tissue specific loss of PTPN2 in IECs. The mice carry loxP sequences flanking exon 3 of the PTPN2 gene and express Cre-recombinase under the control of the vilin promotor. We used these PTPN2 $2^{\text {flox/flox }} x$ VilCre mice to perform acute and chronic dextran sulphate sodium (DSS)-induced colitis. Regarding the preliminary mentioned data, we expected these mice to suffer from more severe inflammation upon DSS treatment.

\section{Materials and Methods}

Mice and Induction of Colitis

Animal experiments were carried out according to Swiss animal welfare laws and were approved by the veterinary authorities of Zurich, Switzerland (Kanton Zürich Gesundheitsdirektion Veterinäramt, approval No. 54/2011). Due to the approval of the veterinary authorities of Zurich, no further approval by an Institutional Animal Care and Use Committee or Ethics Committee was necessary. Mice were housed in a specific pathogenfree facility in individually ventilated cages. Food and water were available ad libitum. Female PTPN2 $2^{\text {flox/flox }}$ (referred to as wt) and PTPN2 ${ }^{\text {flox/flox}}$ VilCre (referred to as knockout, ko) littermates on a C57BL/6J background were used for all experiments. Mice with a bodyweight between 20 and 25 g were randomly divided into 2 DSS and 2 water control groups. Acute colitis was induced by administering 2.5 or $2 \%$ DSS (MP Biomedicals, Illkirch, France) dissolved in drinking water for 7 days. Chronic colitis was induced by 4 cycles of treatment with $1.5 \%$ DSS for 7 days interspersed with 10 days of regular drinking water and a final recovery phase of 3.5 weeks. Body weight and disease activity were determined regularly. At the end of the experiment, colonoscopy was performed in a blinded manner and mice were subsequently sacrificed. Spleen and colon were removed, and colon length and spleen weight were measured. Colon samples were collected for $\mathrm{H} \& \mathrm{E}$ staining at days 3 and 7.

\section{Assessment of Colonoscopy Score in Mice}

Animals were anaesthetised intraperitoneally with 90-120 mg/ kg body weight ketamine (Vétoquinol, Bern, Switzerland) and $8 \mathrm{mg} / \mathrm{kg}$ body weight xylazine (Bayer, Lyssach, Switzerland). Colonoscopy was done as described previously [14]. Briefly, the solid endoscope was introduced per anus with a lubricant (2\% lidocaine) in the sedated mouse. The colon was gently inflated with air. Recording was performed with the Karl Storz Tele Pack Pal 20043020 (Karl Storz Endoskop, Tuttlingen, Germany). Colonoscopy was scored using the murine endoscopic index of colitis severity (MEICS) scoring system as described previously [14]. The following parameters were assessed in detail: (1) thickening of the colon wall (transparent, moderate, marked, non-transparent 0-3 points), (2) changes of the vascular pattern (normal, moderate, marked, bleeding 0-3 points), (3) fibrin visible (none, little, marked, extreme $0-3$ points), (4) granularity of the mucosal sur- 
face (none, moderate, marked, extreme $0-3$ points) and (5) stool consistency (normal solid, still shaped, unshaped, spread 0-3 points). This results in an overall MEICS score range between 0 and 15.

\section{Assessment of Histological Score in Mice}

Histological scoring for inflammatory infiltration and epithelial cell damage were performed on H\&E-stained sections of the most distal $1 \mathrm{~cm}$ of the mouse colon as described previously [15]. Score for epithelial damage: 0 , normal morphology; 1 , loss of goblet cells; 2 , loss of goblet cells in large areas; 3 , loss of crypts; 4 , loss of crypts in large areas. Score for infiltration: 0, no infiltrate; 1, infiltrate around crypt base; 2 , infiltrate reaching to L. muscularis mucosae; 3, extensive infiltration reaching the L. muscularis and thickening of the mucosa with abundant oedema; 4 , infiltration of the L. submucosae. The total histological score represents the sum of the scores for epithelial damage and infiltration. Histological examination was performed by an independent, blinded investigator.

\section{IEC and DNA Isolation from Colon Specimens}

After removing the most distal $3 \mathrm{~cm}$ of colon for other purposes, the remaining part of the colon was cut lengthwise, freed from residual faeces and cut into small pieces. To loosen IEC, the slices were transferred to Hank's balanced salt solution (HBSS; PAA, Linz, Austria) supplemented with $2 \mathrm{mM}$ of EDTA and stirred for $30 \mathrm{~min}$ at $37^{\circ} \mathrm{C}$. Mucosal pieces were removed by passing the slurry over a coarse mesh $(400 \mu \mathrm{m}$; Carl Roth $\mathrm{GmbH}$, Karlsruhe, Germany). The sieve residue was flushed back to the tube with fresh HBSS, vigorously shaken 10 times and vortexed for $1 \mathrm{~min}$. The shake and vortex step was repeated 2 times resulting in 30 times shaking and 3 min of vortexing. Mucosal pieces were partitioned again from detached cells by filtration through mesh. Through fraction was collected and centrifuged at $400 \mathrm{~g}$ for $7 \mathrm{~min}$. Supernatant was discarded and the cell pellet was resuspended in PBS. The supernatant was removed and samples were frozen in liquid nitrogen and stored at $-80^{\circ} \mathrm{C}$ until DNA, RNA or protein isolation. The pelleted IECs were thawed and DNA was isolated using the DNA Mini Kit (Qiagen, Düsseldorf, Germany) based on the manufacturer's blood and body fluid spin protocol.

\section{RNA Isolation, RT-PCR and Quantitative PCR}

To isolate RNA, IEC were isolated as described above and resuspended in $350 \mu \mathrm{l}$ RLT buffer (Qiagen, Venlo, Netherlands). For RNA isolation from colon tissue, $1 \mathrm{~cm}$ long colon pieces were lysed in $350 \mu \mathrm{RLT}$ buffer in gentleMACS tissue disrupter tubes (Miltenyi Biotec, Bergisch Gladbach, Germany) by adhering to the manufacturer's instructions. Total RNA was isolated using RNeasy Plus Mini Kit (Qiagen) according to manufacturer's instructions. RNA concentration was measured by absorbance at 260 and $280 \mathrm{~nm}$. Complementary DNA (cDNA) synthesis was performed using a high-capacity cDNA reverse transcription kit (Life Technologies, Foster City, Calif., USA) following the manufacturer's instructions. Real-time polymerase chain reaction (PCR) was performed using FAST qPCR MasterMix for Taqman assays (Life Technologies) on a Fast HT7900 Real-Time PCR system using SDS software (Life Technologies). Measurements were performed in triplicate, mouse $\beta$-actin was used as endogenous control, and results were analyzed by $\Delta \Delta \mathrm{CT}$ method. The real-time PCR con- tained an initial enzyme activation step $\left(5 \mathrm{~min}, 95^{\circ} \mathrm{C}\right)$ followed by 45 cycles consisting of a denaturing $\left(95^{\circ} \mathrm{C}, 15 \mathrm{~s}\right)$ and an annealing/ extending $\left(60^{\circ} \mathrm{C}, 1 \mathrm{~min}\right)$ step. The used gene expression assays were all obtained from Life Technologies.

\section{Protein Isolation and Western Blotting}

IEC isolated as described above, or $1 \mathrm{~cm}$ long colon pieces taken $1 \mathrm{~cm}$ proximally from the rectum, were homogenized in M-PER cell lysis buffer (Thermo Fisher Scientific, Waltham, Mass., USA) using gentleMACS tissue disrupter tubes (Miltenyi Biotec) according to the manufacturer's instruction. The homogenates were lysed for $30 \mathrm{~min}$ on ice and centrifuged for $10 \mathrm{~min}$ to remove cell debris. Equal amounts of protein from each lysate were loaded on polyacrylamide gels and after separation by gel-electrophoresis blotted onto nitrocellulose membranes. Membranes were incubated over night with anti-mouse PTPN2 antibody (Merck Millipore, Billerica, Mass., USA), washed 3 times with washing buffer (tris buffered saline, 3\% milk) before incubation with HRP-coupled anti-mouse secondary antibody (Santa Cruz Biotechnologies, Dallas, Tex., USA) for $2 \mathrm{~h}$. Immunoreactive proteins were detected with a Fusion Solo S imager (Vilber Lourmat, Eberhardzell, Germany) using an enhanced chemiluminescence detection kit (GE Healthcare, Little Chalfont, UK).

\section{Immunohistochemistry}

Immunohistochemical studies were performed on formalinfixed, paraffin-embedded tissue specimen using a peroxidasebased method with diaminobenzidine (DAB) chromogen. Tissue samples were incubated with xylol and descending concentrations of ethanol. Antigen retrieval was performed using citrate buffer, pH 6.0 (DAKO, Glostrup, Denmark) for $30 \mathrm{~min}$ at $98^{\circ} \mathrm{C}$. Endogenous peroxidases were deleted by incubation with $0.9 \%$ hydrogen peroxide for $15 \mathrm{~min}$ at room temperature (RT) and blocking was performed using $3 \%$ bovine serum albumin overnight in a wet chamber. Sections were stained for $2 \mathrm{~h}$ at RT with an appropriate concentration of mouse anti-PTPN2 antibody (Merck Millipore). HRP-labelled secondary anti-mouse IgG-antibody (Santa Cruz Biotechnology) was applied for $1 \mathrm{~h}$ at RT and antibody binding visualized by a liquid $\mathrm{DAB}+$ substrate chromogen system (DAKO). Then samples were counterstained with haematoxylin, dehydrated in ascending concentrated ethanol and xylol solutions and finally mounted. Microscopic assessment was done using an AxioCam HRc (Zeiss, Jena, Germany) on a Zeiss Axio Imager. Z2 microscope (Zeiss) with AxioVision release 4.8.2 software (Zeiss).

\section{Genotyping}

Digestion buffer ( $25 \mathrm{~mm} \mathrm{NaOH}, 0.2 \mathrm{~mm}$ EDTA) was added to ear biopsies and incubated for $1 \mathrm{~h}$ at $98^{\circ} \mathrm{C}$. An equal amount of neutralisation buffer ( $40 \mathrm{~mm}$ Tris- $\mathrm{HCl}, \mathrm{pH}$ 5.5) was added and $1 \mu \mathrm{l}$ was used for genotyping VilCre PCR to assess the presence of the cre-recombinase. DNA isolated from IECs from colon specimens was used to check if cre-mediated recombination took place via genotyping PCR. Primer sequences VilCre PCR: VilCre_forward: GTGTGGGACAGAGAACAAACC,VilCre_reverse:ACATCTTC AGGTTCTGCGGG, Contr_forward: CAAATGTTGCTTGTCT GGTG, Contr_reverse: GTCAGTCGAGTGCACAGTTT; primer sequences for PTPN2 recombinant allele: primer_forward: GCC AAGAGACAGTGGAAAGAGAG, Primer_reverse: ACTGCAA AACCATAACTGGGC. 


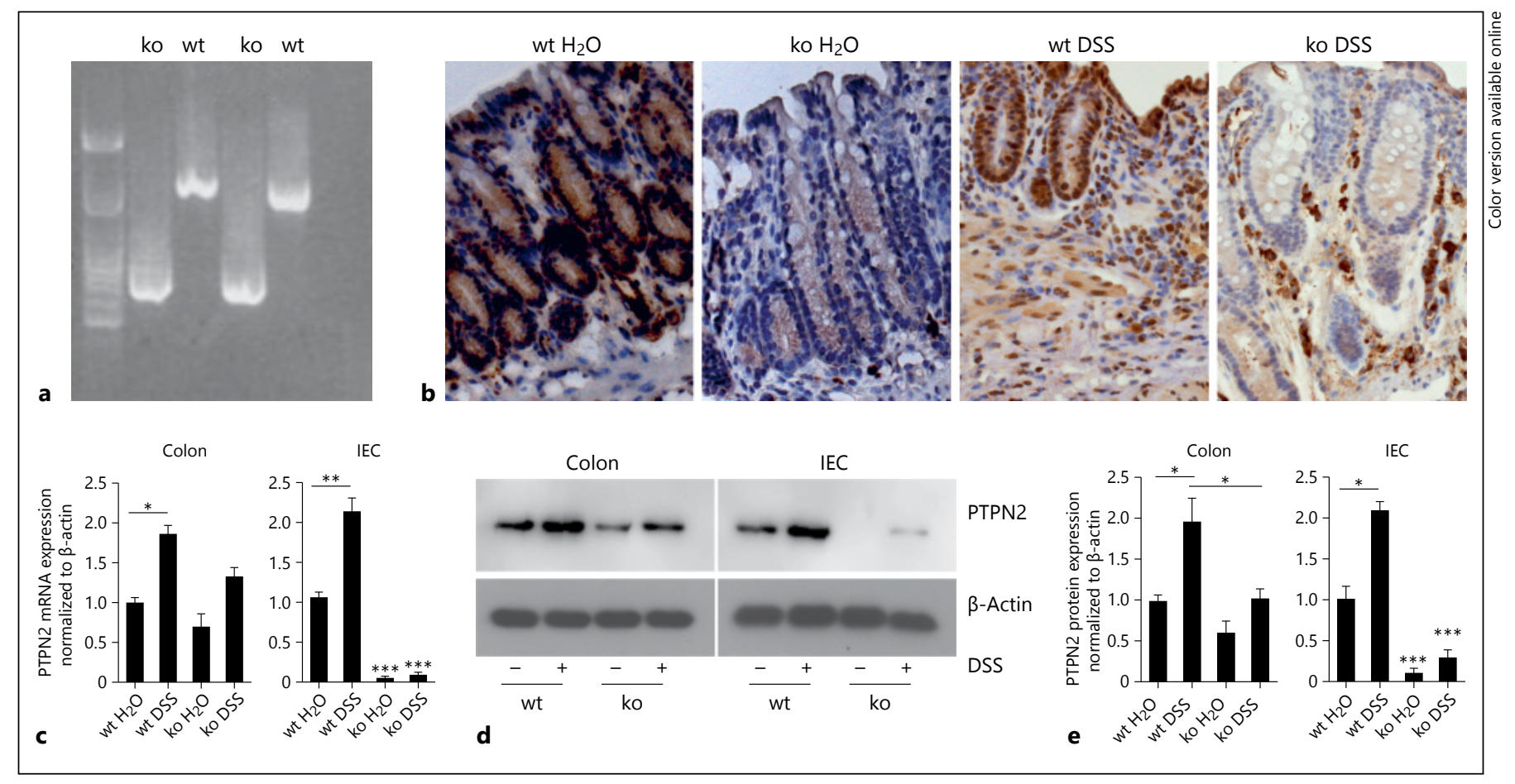

Fig. 1. Loss of PTPN2 in IECs of PTPN2xVilCre mice. a IECs were isolated from colon specimens of PTPN2 $2^{\text {flox/flox }} \times \mathrm{VilCre}(\mathrm{ko})$ and PTPN2 flox/flox $(w t)$ mice. DNA was isolated from these IECs and used for genotyping PCR. Amplified PCR products were run in a $3 \%$ agarose gel and $100 \mathrm{bp}$ DNA ladder was used to estimate amplicon lengths. b-d wt and ko mice were treated for 7 days with

\section{Wound Healing Assay}

For the in vitro wound-healing assay, HT-29 cells (DSMZ, Germany) were transfected with non-targeting control, or PTPN2 specific siRNA (Life Technologies) using an Amaxa nucleofector (Lonza, Basel, Switzerland) prior to seeding at a density of $1 \times 10^{6}$ cells $/ \mathrm{ml}$ in 6 well plates. Cells were grown for 2 days to reach full confluency before generating a scratch using a pipette tip. Closure of the scratch area was monitored using a bright field microscope (Zeiss).

The in vivo wound-healing model was performed as described [16]: during endoscopy, mucosal wounds were induced mechanically using a biopsy forceps with a diameter of 3 French (equals $1 \mathrm{~mm}$ ). Subsequently, wound healing was monitored by daily endoscopy and quantified by measurement of the residual wound area using ImageJ image processing software. The individual wound closure over time was calculated by dividing actual wound area/initial wound area and is presented as percentage.

\section{Results}

Mice with Tissue Specific Loss of PTPN2 in IECs

To determine the role of the IBD susceptibility gene PTPN2 in the intestinal epithelium in vivo, we gener-
$2.5 \%$ DSS to induce acute colitis and terminal colon tissue was stained for PTPN2 by IHC (b); colon lysates or isolated IECs were analysed for PTPN2 mRNA expression by RT-PCR (c); and PTPN2 protein levels by western blotting $(\mathbf{d}, \mathbf{e})$. Asterisks denote significant differences $\left({ }^{*} \mathrm{p}<0.05,{ }^{* *} \mathrm{p}<0.01,{ }^{* * *} \mathrm{p}<0.001\right.$, Neuwmann Keul's test).

ated mice that exhibited a tissue-specific loss of PTPN2 in IECs (PTPN2 $2^{\text {flox/flox }} x$ VilCre mice). To confirm crerecombinase mediated recombination of the PTPN2 allele, PTPN $2^{\text {flox/flox }}$ and PTPN $2^{\text {flox/flox }} x$ VilCre mice were sacrificed and IECs were isolated from colon specimens. DNA was isolated from these IECs and used for genotyping PCR. In fact, figure 1 shows that cre-recombinase was active in PTPN $2^{\text {flox/flox}} \mathrm{xVilCre}$ mice and mediated conversion of the PTPN2 allele in IECs. Further, immunohistochemistry (IHC) confirmed loss of PTPN2 protein expression in IEC in health and after induction of acute colitis using 2.5\% DSS for 7 days (fig. 1b). While only a slight reduction of PTPN2 mRNA expression was found in the colon, its expression was lost in isolated IEC (fig. 1c). Similar results were obtained when analysing PTPN2 protein expression by western blot (fig. 1d). Of note, PTPN2 expression was enhanced in the colon and in IEC of DSS-treated PTPN2 $2^{\text {flox/flox }}$ animals, but not in PTPN2 $2^{\text {flox/flox }} x$ VilCre mice (fig. 1c, d). 


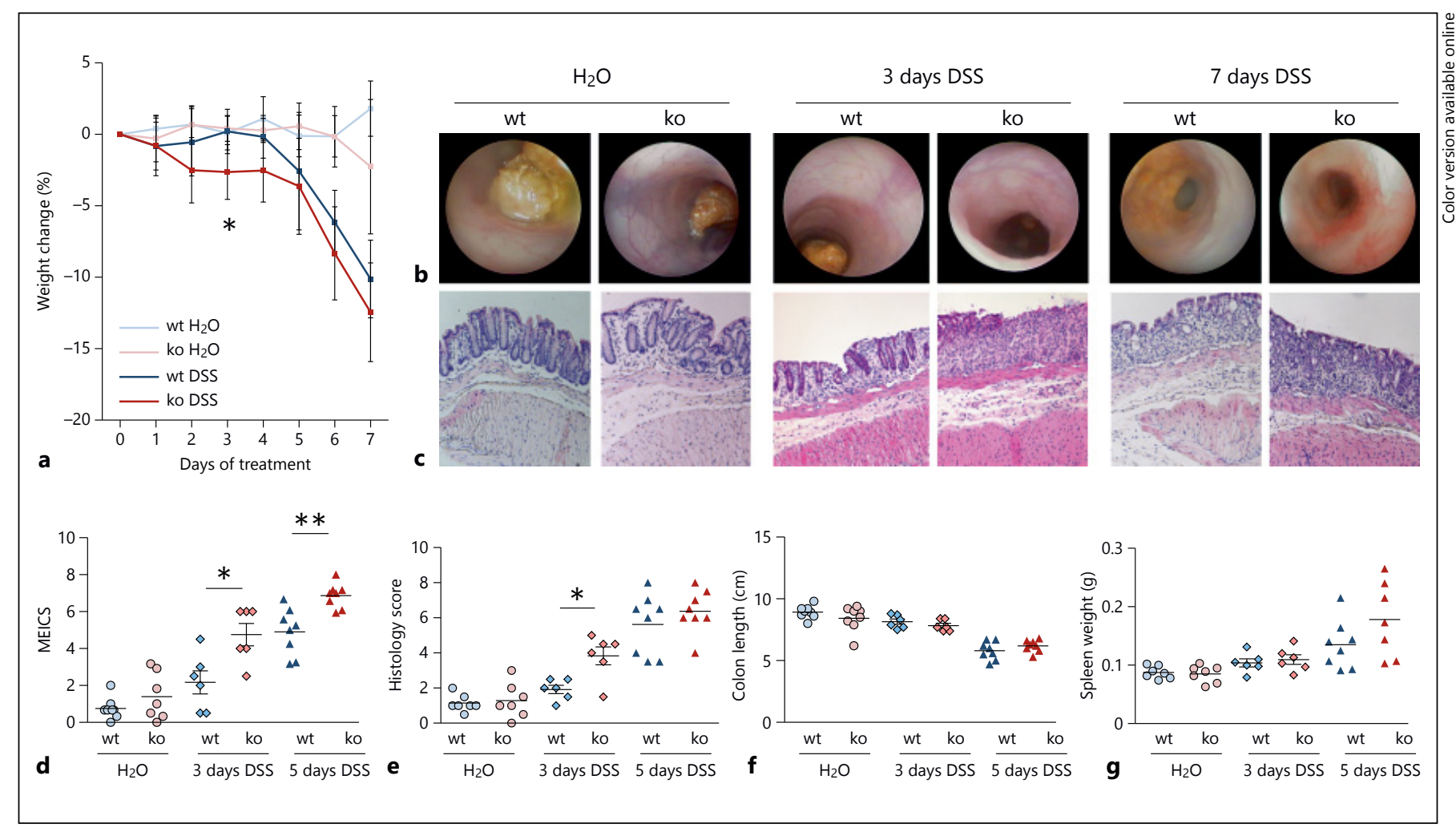

Fig. 2. PTPN2xVilCre mice tended to exhibit more severe inflammation in acute colitis using $2.5 \%$ DSS. Female PTPN $2^{\text {flox } / \text { flox }} x$ VilCre (ko) and their PTPN2 $2^{\text {flox/flox }}$ (wt) littermates received $2.5 \%$ DSS for 7 days and body weight was measured daily. To assess colitis severity, colonoscopy was performed at days 3 and 7 of the experiments, and mice were sacrificed at day $3(n=6)$ and day $7(n=8)$ to assess histological changes. a Weight changes in percent indicate early onset of weight loss in PTPN2xVilCre mice, data are presented as mean \pm SD. b Representative pictures from colonoscopy at days 3 and 7 indicate more severe disease in ko animals, while H\&E-stained sections from the distal colon reveal no difference (c). d Accordingly, MEICS show more severe DSS-induced inflammation in PTPN2xVilCre mice, but quantification of H\&E-stained sections (e) reveal no difference. Colon length (f) and Spleen weight $(\mathbf{g})$ were measured but both parameters were not influenced by genotype. $\mathbf{d}-$ g The values for individual mice, horizontal bars indicate mean, Mann-Whitney $U$ test was used for statistical analysis, ${ }^{*} \mathrm{p}<0.05$, ** $\mathrm{p}<0.001$.

\section{PTPN2xVilCre Mice Tended to Suffer More from}

Acute Colitis Induced by 2.5\% DSS

The DSS-induced colitis, a model of mucosal inflammation to study IBD pathogenesis, was used for experimental approaches. In a first acute colitis experiment we administered $2.5 \%$ DSS to PTPN2 ${ }^{\text {flox/flox }} \mathrm{xV}$ Vilcre (PTPN2xVilCre) mice and their PTPN2 $2^{\text {flox/flox }}$ (wt) littermates for 7 days. The DSS treatment induced severe weight loss in both groups (fig. 2a). At the end of the experiment there was no difference in weight loss between genotypes but the onset of weight loss was earlier in the DSS-treated PTPN2xVilCre animals compared to their DSS-treated wt littermates, leading to a significant difference at day 3 (fig. 2a). Colonoscopy at day 3 as well as at the end of the experiment revealed significant difference between DSS groups, with PTPN2xVilCre mice showing signs of a more severe inflammation (fig. 2b). Further, we scored H\&E-stained colon slides for infiltration and epithelial damage. While histology at day 3 revealed slightly enhanced colitis in PTPN2xVilCre animals, no difference could be detected at day 7 . Nevertheless, the score revealed a very severe inflammation at day 7 even in the DSS-treated wt group with one mouse reaching a maximum score of 8. Colitis induction further led to a marked shortening of the colon at day 7 , but the magnitude was not influenced by PTPN2 deficiency in IEC (fig. 2c). Also, at day 7 the spleen weight was increased indicative of a very severe DSS-induced inflammation in both, wt and PTPN22xVilCre animals (fig. 2d). Therefore, it seemed plausible that wt animals already showed such a pronounced inflammation that it was not possible for the PTPN2xVilCre group to exceed their inflammation. 


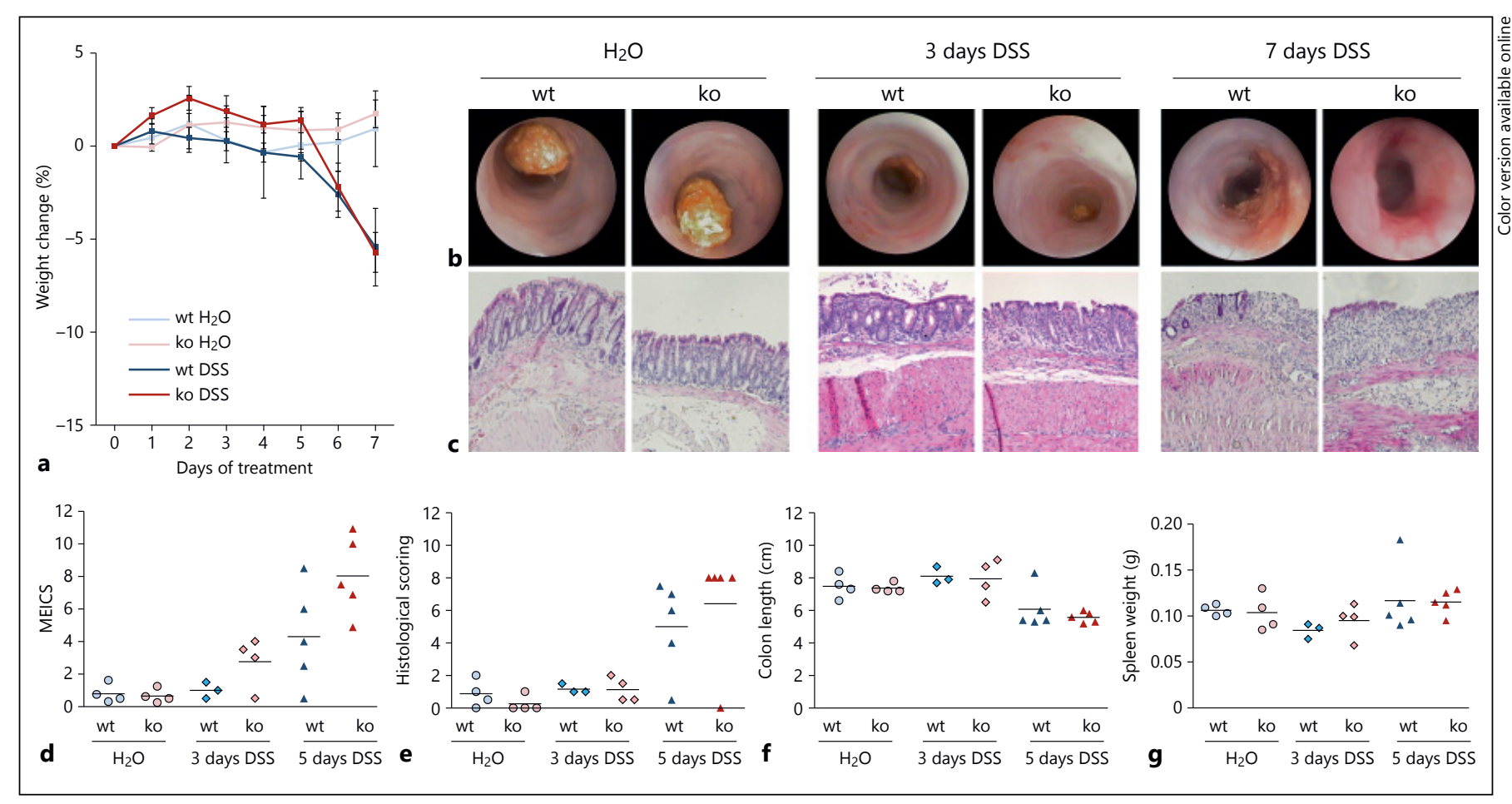

Fig. 3. No differences in inflammation severity in acute colitis using $2 \%$ DSS. Female PTPN2 $2^{\text {flox/flox }} x$ VilCre $(\mathrm{ko})$ and their PTPN2 $2^{\text {flox/flox }}$ (wt) littermates received 2\% DSS for 7 days $(n=4 / 5)$. Body weight was measured daily and (a) percental weight changes are presented as mean \pm SD. b-e At day $3(n=3)$ and day $7(n=4 / 5)$ colonoscopy was performed and MEICS was used to determine inflammation severity. On these days, mice were also sacrificed and terminal

Hence, we decided to lower the DSS concentration in a second acute DSS colitis experiment, to investigate whether the differences between wt and PTPN2xVilCre mice would be more pronounced during a more moderate colitis.

\section{PTPN2 Deficiency in IECs Did Not Influence}

Inflammation in Acute Colitis Induced by $2 \%$ DSS

A milder colitis was induced by the administration of $2 \%$ DSS for 7 days and the resulting weight curve is shown in figure 3a. Regarding weight loss, we did not detect any differences between equally treated groups during the whole experiment (fig. $3 \mathrm{a}$ ). In contrast to the $2.5 \%$ DSS colitis, the early onset of weight loss in the DSS-treated PTPN2xVilCre mice did not reoccur when using less DSS. Also, colonoscopy and histologic assessment of microscopic disease severity as well as the respective scores did not reveal significant differences between genotypes, neither at day 3 nor at day 7 of the experiment (fig. 3b-e). There was only a minor trend towards a more severe in- colon assessed for histological changes. b Representative colonoscopic pictures; c representative pictures from H\&E-stained sections of the terminal colon; d MEICS; e histological scores show no influence of PTPN2 deficiency. Colon length (f) and spleen weight (g) was measured but both parameters were not altered by genotype. $\mathbf{b}-\mathbf{g}$ The values for individual mice, horizontal bars indicate mean, Mann-Whitney U test was used for statistical analysis. flammation in PTPN2 ko animals. The DSS treatment resulted in the shortening of the colon and increased spleen weight induced by inflammation but was independent of the genotype (fig. 3f, g). Interestingly, the variation within DSS groups was extremely high and some animals even showed normal healthy mucosa.

\section{PTPN2xVilCre Mice Tended to Suffer Less from Chronic DSS Colitis}

To see whether a prolonged inflammation would reveal a phenotype in PTPN2xVilCre mice, we performed a chronic colitis experiment by 4 cycles of DSS treatment and a final recovering phase of 4 weeks. The weight curve (fig. 4a) revealed no significant differences. Surprisingly, during acute phases, the weight loss of PTPN2xVilCre mice tended to be less pronounced compared to their wt littermates. This unexpected trend was also visible in the colonoscopic scoring: DSS-treated PTPN2 ko animals showed significantly less severe inflammation compared to their wt littermates (fig. 4b). This unexpected differ- 


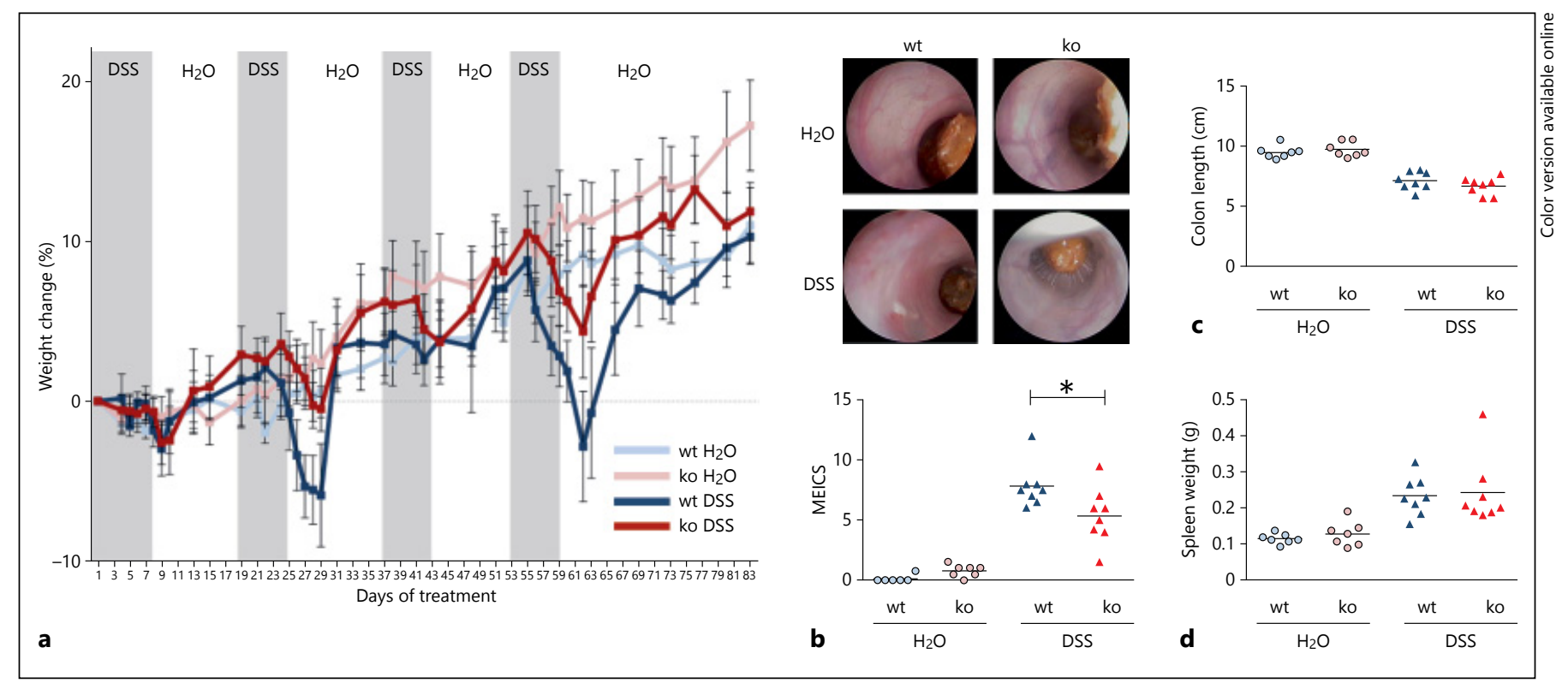

Fig. 4. PTPN2xVilCre mice tended to exhibit less severe inflammation in chronic DSS colitis. Female PTPN2 ${ }^{\text {flox } / \text { flox }} x$ VilCre (ko) and their PTPN2 $2^{\text {flox/flox }}(\mathrm{wt})$ littermates received 4 cycles of DSS treatment and were allowed to recover afterwards for 3.5 weeks $(\mathrm{n}=7 / 8)$. Body weight was measured regularly and (a) percental weight changes are presented as mean \pm SD. Colonoscopy was done at the end of the experiment and MEICS was used to deter-

ence was not reflected in the histological scoring of H\&E-stained colon slides (fig. 5a). Histology did not even show a trend but rather the same degree of mucosal damage in both DSS groups. However, H\&E-stained sections showed epithelial abnormalities in DSS-treated mice that were not observed in acute colitis experiments. Several aberrant crypts of different shapes formed clusters that were clearly distinguished from the surrounding tissue (fig. 5b). Aberrant crypt foci like that are known to be a consequence of aberrant proliferation upon exposure to carcinogens and could develop into colon cancer $[17,18]$. Importantly, the aberrant crypt foci were significantly more frequent in DSS-treated PTPN2xVilCre mice (fig. 5b). In one mouse, abnormal foci even penetrated the lamina muscularis and aberrant crypts were found in the submucosa.

\section{IEC-Specific Loss of PTPN2 Promotes IEC}

Proliferation and Wound Healing

So far we observed that PTPN2xVilCre mice suffered from a more severe colitis at day 3 of $2.5 \%$ DSS treatment but seemed to be able to compensate for this early weight loss. Further, PTPN2xVilCre mice tended to suffer less in mine inflammation severity. b Representative colonoscopic pictures and MEICS show less severe inflammation in mice with PTPN2 deficiency in IECs. Mice were sacrificed; colon length (c) and spleen weight (d) were measured but both parameters were not altered by genotype. b-d The values for individual mice, horizontal bars indicate mean, Mann-Whitney $U$ test was used for statistical analysis, ${ }^{*} \mathrm{p}<0.05$. chronic DSS-induced colitis, and showed aberrant crypt proliferation. Hence, we hypothesized that IEC-specific loss of PTPN2 might in general influence epithelial proliferation and wound-healing mechanisms. To address this hypothesis, we first used an in vitro model for proliferation and wound healing, where a scratch is induced in a confluent monolayer of HT29 IEC and the closure of the scratch is monitored. While in control siRNA transfected cells the scratch width was still $30-50 \%$ of the initial distance after $48 \mathrm{~h}$, transfection with PTPN2 specific siRNA resulted in almost complete closure of the gap in the same time period (5-15\% of initial width; fig. 6a). This indicates that loss of PTPN2 in epithelial cells might indeed affect proliferation and wound closure. To further assess this hypothesis, we next stained intestinal specimen from wt and PTPN2xVilCre mice from the 2.5\% DSS colitis experiment (day 7) for Ki67, a general marker for cell proliferation. In wt animals, only a few Ki67 positive cells were detected, although Ki67 staining was increased upon DSS treatment. In PTPN2xVilCre animals, there were elevated levels of Ki67+ cells even in the healthy colon, and numbers further increased upon colitis induction (fig. 6b). To finally address whether in vivo wound healing is af- 


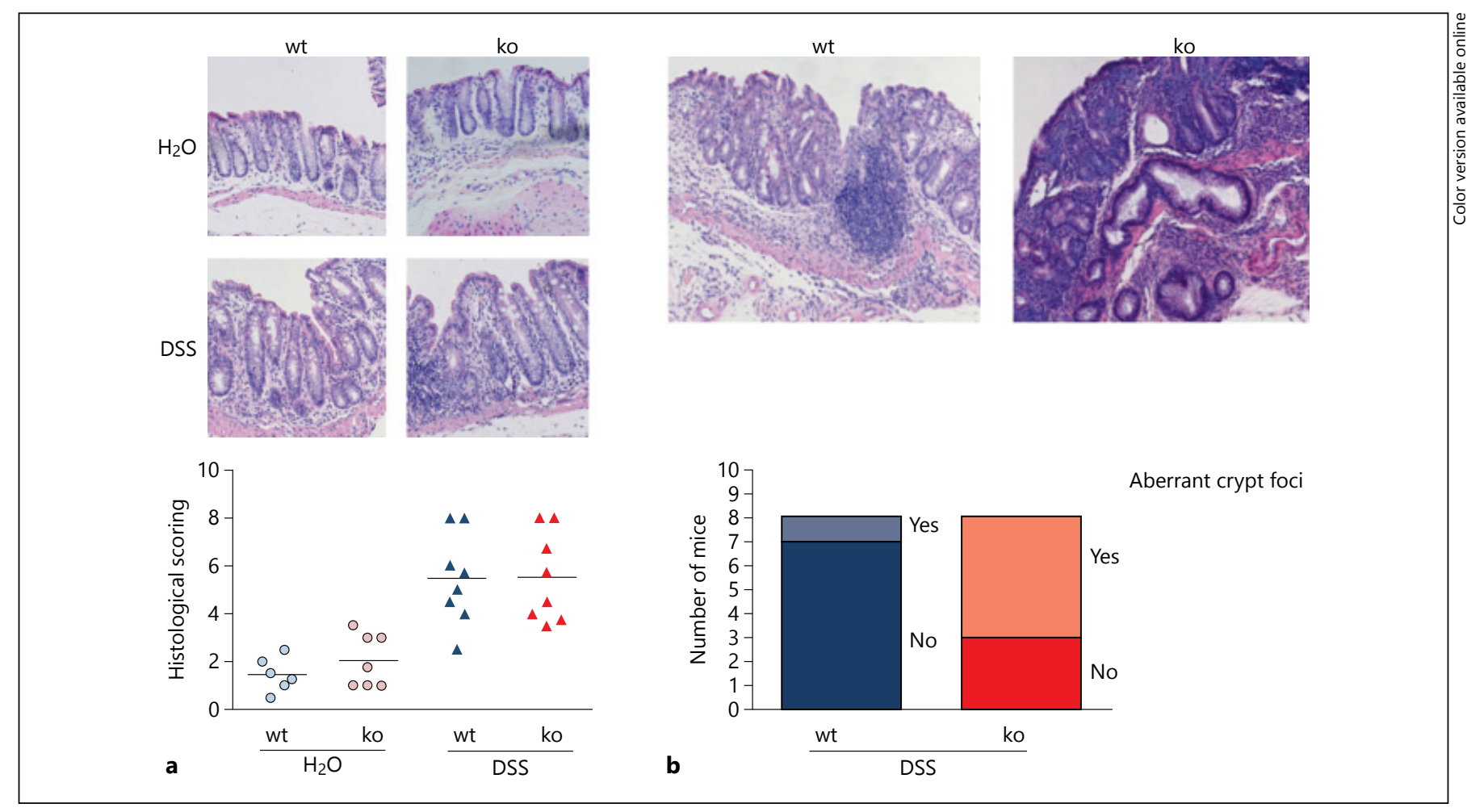

Fig. 5. PTPN2xVilCre mice developed more frequently aberrant crypt foci in chronic DSS colitis. To induce chronic colitis PTPN2 ${ }^{\text {flox/flox }} x$ VilCre (ko) and their PTPN2 $2^{\text {flox/flox }}$ (wt) littermates received 4 cycles of DSS treatment and were allowed to recover afterwards for 3.5 weeks $(n=7 / 8)$. At the end of the experiment, mice were sacrificed and distal colon slides were used for $\mathrm{H} \& \mathrm{E}$

fected upon loss of PTPN2 in IEC, we used an in vivo model for wound healing, where a small epithelial wound is mechanically induced in the colon epithelium and wound closure measured daily by endoscopy. In this model, we found a strong trend towards faster wound closure in PTPN2xVilCre mice when compared to their wt littermate controls (fig. 6c).

\section{Discussion}

In the first acute colitis experiments, we observed an early onset of weight loss in DSS-treated PTPN2xVilCre mice compared to their wt littermates and colonoscopy showed significantly more severe inflammation in those animals. In a second experiment using a lower DSS concentration, these differences did not reoccur. In a chronic DSS colitis, mice with PTPN2 deficiency in IECs tended to less pronounced weight loss during acute phases and colonoscopy revealed less severe inflammation by the staining and scored for epithelial damage and infiltration. a Representative pictures and graph presenting histological score exhibit no difference between genotypes. Graph shows values for individual mice, horizontal bars indicate mean. b Aberrant crypt foci were detected more frequently in H\&E-stained slides of distal colon in ko mice.

end of the experiment. In all 3 experiments, histological scoring did not show any differences between genotypes. The fact that histological scoring is regarded as the gold standard in evaluation of inflammation severity debilitates the differences we found in weight loss or colonoscopy. Obviously, in the context of DSS colitis PTPN2 plays only a minor role in IECs during inflammation.

These findings are apparently in contrast to cell culture studies that demonstrated the importance of PTPN2 expression in IECs for barrier function, secretion of pro-inflammatory cytokines and autophagy $[8,11-13]$, what seems to have no apparent in vivo effects. The mechanism of DSS-induced inflammation involves erosion of the epithelial barrier $[19,20]$, generating breaches where pathogens and commensals can enter and elicit a strong immune reaction [21]. During intestinal inflammation, the ability of IECs to proliferate and regenerate is crucial for the restoration of the integrity of the mucosal barrier and termination of inflammation [22]. Therefore, increased IEC proliferation and more efficient wound healing might explain why 


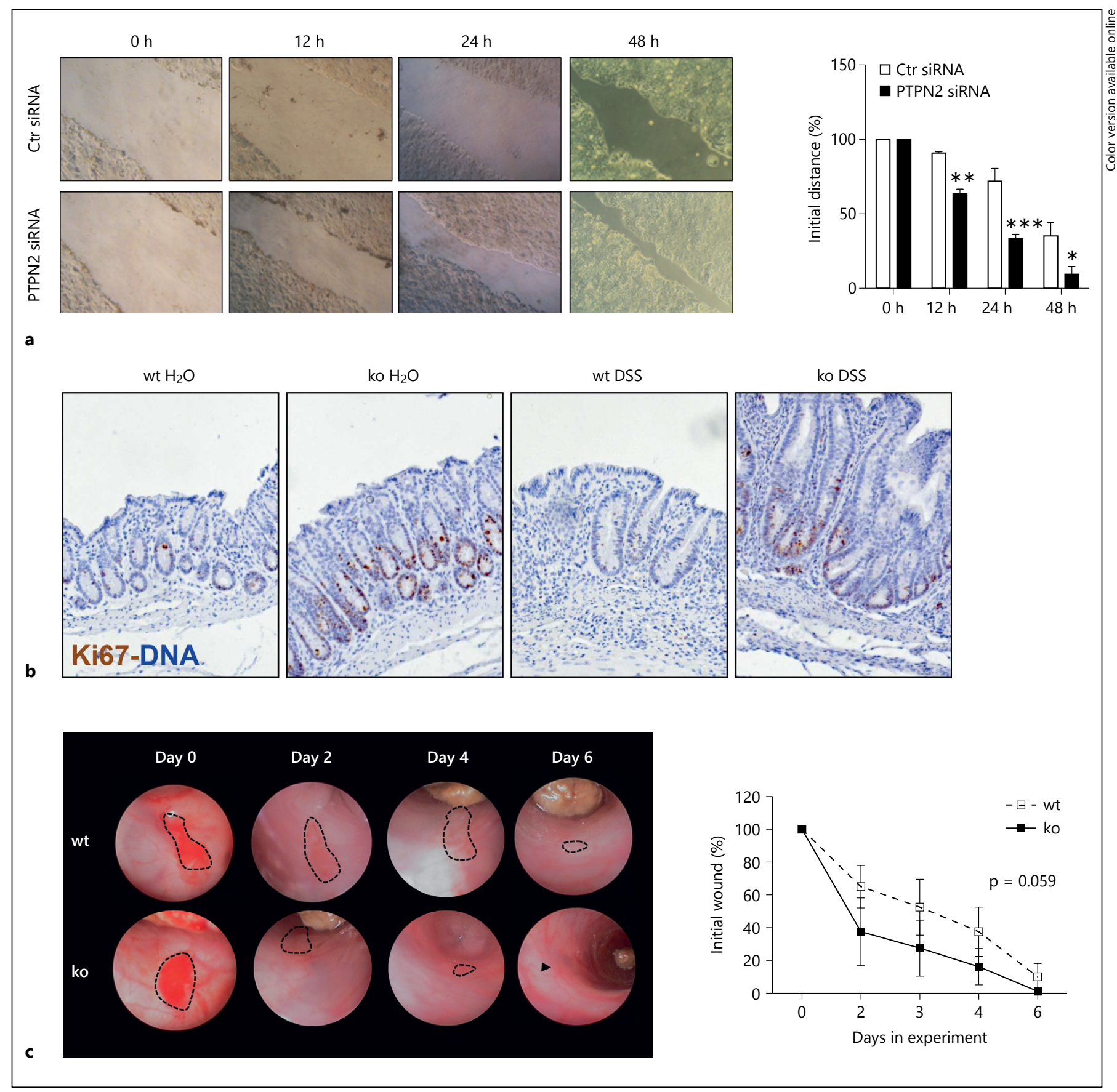

Fig. 6. Loss of PTPN2 in IEC promotes proliferation and intestinal wound closure. a HT29 cells were transfected with non-targeting control or with PTPN2-specific siRNA and grown to confluency. A scratch was induced and closure of the scratch monitored at indicated time-points. Representative pictures and quantification of the relative scratch width indicate faster closure in PTPN2-deficient HT-29. b Female PTPN2 ${ }^{\text {flox/flox }} x$ VilCre (ko) and their
PTPN2 $2^{\text {flox/flox }}(\mathrm{wt})$ littermates received $2.5 \%$ DSS for 7 days. Sections from the distal colon were stained for the proliferation marker Ki67, and this reveals increased IEC proliferation in ko animals (c). Mucosal wounds were introduced in ko and wt littermates and wound closure monitored by daily endoscopy. Representative pictures and statistical analysis of relative wound closure show a strong trend toward increased wound healing in ko animals. 
IEC-specific deletion of PTPN2 does not result in increased pathology upon DSS treatment. Although lack of PTPN2 in IEC might result in defective epithelial barrier functions [11] or enhanced secretion of pro-inflammatory cytokines $[23,24]$, increased proliferation can obviously compensate for those effects. EGFR is among the known targets of PTPN2 [5], which might explain the increased proliferation in PTPN2-deficient IEC. Also ERK and p38 - both targets of PTPN2 in IEC $[23,25]$ - have been implicated in IEC proliferation [26, 27]. However, further analysis would be necessary to address the molecular mechanisms as to how loss of PTPN2 mediates increased proliferation.

Further, DSS-induced inflammation is mediated by the interplay of many different cells types, not only IECs but also cells of the innate and adaptive immune system. When PTPN2 function is lost in IECs, the body seems to be able to compensate this shortcoming. However, in vivo studies using other tissue-specific PTPN2 knock out mouse strains have already demonstrated the importance of this phosphatase for the development of $\mathrm{CD} 4^{+}$cells [28] but also for TCR signalling and lymphopenia-induced proliferation in $\mathrm{CD}^{+}$cells $[29,30]$. Apparently, loss of PTPN2 in the T cell compartment cannot be counterbalanced and leads to strong phenotypes. This is not surprising regarding the fact that PTPN2 has the highest expression in $\mathrm{T}$ cells and was originally discovered in a screening of a human T cell cDNA library [31].

After induction of chronic colitis, we detected epithelial aberrant crypt foci in HE-stained sections of the distal colon in DSS-treated animals. This kind of epithelial transformations has been observed in other studies as a result of exposure to carcinogens and could develop into colon cancer $[17,18]$. Those abnormalities were present in most knockout animals but only in one wt mouse. This again fits to the increased proliferative capacity of IEC we observed upon loss of PTPN2. Further, the PTPN2 target EGFR is involved in tumour progression, and might be involved in the formation of aberrant crypts in our mice. In conclusion, these aberrant crypt foci further indicate that loss of PTPN2 in IEC might promote tumour progression. However, more detailed analysis will be necessary to address this hypothesis.

\section{Funding}

This research was supported by a grant from the European Crohn's and Colitis Organisation to M.S., Fonds zur Förderung des akademischen Nachwuchses of the Zürcher Universitätsverein to M.S., a research grant from the Swiss Philanthropy Foundation to M.S. and G.R., a research credit from the University of Zurich to M.S., research grants from the Swiss National Science Foundation to M.S. (grant Nos. 314730-146204 and CRSII3_154488/1), to G.R. (grant No. 310030-120312) and the Swiss IBD Cohort (grant No. 3347CO-108792) and by a grant from the Zurich Center for Integrative Human Physiology of the University of Zurich to M.S.

\section{Disclosure Statement}

The authors declare no competing interests.

\section{Author Contributions}

Animal breeding (A.F.-T., S.H.K., M.R.S.), application for animal license (I.F.-W., M.R.S., M.S.), animal care and recording of weight curves (S.H.K.), sacrifice of mice and sampling (S.H.K., I.L., A.G., T.R., C.G., K.A.), colonoscopy (M.R.S., S.H.K.), determination of MEICS (M.R.S., S.H.K.), histological scoring (I.L., S.H.K.), isolation of DNA from murine IECs (S.H.K.), study design (M.S., G.R., S.H.K.), manuscript writing (S.H.K., M.S.), figure design (S.H.K., M.S.); all authors wrote, corrected and approved the manuscript.

\section{References}

1 Cho JH, Brant SR: Recent insights into the genetics of inflammatory bowel disease. Gastroenterology 2011;140:1704-1712.

2 Todd JA, et al: Robust associations of four new chromosome regions from genome-wide analyses of type 1 diabetes. Nat Genet 2007; 39:857-864.

3 Smyth DJ, et al: Shared and distinct genetic variants in type 1 diabetes and celiac disease. N Engl J Med 2008;359:2767-2777.

4 Wellcome Trust Case Control Consortium: Genome-wide association study of 14,000 cases of seven common diseases and 3,000 shared controls. Nature 2007;447:661-678.
5 Doody KM, Bourdeau A, Tremblay ML: T-cell protein tyrosine phosphatase is a key regulator in immune cell signaling: lessons from the knockout mouse model and implications in human disease. Immunol Rev 2009;228:325-341.

6 Simoncic PD, et al: The T cell protein tyrosine phosphatase is a negative regulator of janus family kinases 1 and 3. Curr Biol 2002;12: 446-453.

7 Aoki N, Matsuda T: A nuclear protein tyrosine phosphatase TC-PTP is a potential negative regulator of the PRL-mediated signaling pathway: dephosphorylation and deactivation of signal transducer and activator of tran- scription $5 \mathrm{a}$ and $5 \mathrm{~b}$ by TC-PTP in nucleus. Mol Endocrinol 2002;16:58-69.

8 Scharl M, et al: Protein tyrosine phosphatase $\mathrm{N} 2$ regulates $\mathrm{TNF} \alpha$-induced signalling and cytokine secretion in human intestinal epithelial cells. Gut 2011;60:189-197.

9 You-Ten KE, et al: Impaired bone marrow microenvironment and immune function in $\mathrm{T}$ cell protein tyrosine phosphatase-deficient mice. J Exp Med 1997;186:683-693.

10 Heinonen $\mathrm{KM}$, et al: T-cell protein tyrosine phosphatase deletion results in progressive systemic inflammatory disease. Blood 2004; 103:3457-3464. 
11 Scharl M, et al: Protection of epithelial barrier function by the Crohn's disease associated gene protein tyrosine phosphatase N2. Gastroenterology 2009;137:2030-2040.e5.

12 McCole DF: Regulation of epithelial barrier function by the inflammatory bowel disease candidate gene, PTPN2. Ann N Y Acad Sci 2012;1257:108-114.

13 Scharl M, et al: Protein tyrosine phosphatase nonreceptor type 2 regulates autophagosome formation in human intestinal cells. Inflamm Bowel Dis 2012;18:1287-1302.

14 Becker C, Fantini MC, Neurath MF: High resolution colonoscopy in live mice. Nat Protoc 2006;1:2900-2904.

15 Obermeier F, et al: Interferon-gamma (IFNgamma)- and tumour necrosis factor (TNF)induced nitric oxide as toxic effector molecule in chronic dextran sulphate sodium (DSS)induced colitis in mice. Clin Exp Immunol 1999;116:238-245.

16 Neurath MF, et al: Assessment of tumor development and wound healing using endoscopic techniques in mice. Gastroenterology 2010;139:1837-1843.e1.

17 Lam LK, Zhang J: Reduction of aberrant crypt formation in the colon of CF1 mice by potential chemopreventive agents. Carcinogenesis 1991;12:2311-2315.
18 Tudek B, Bird RP, Bruce WR: Foci of aberrant crypts in the colons of mice and rats exposed to carcinogens associated with foods. Cancer Res 1989;49:1236-1240.

19 Wirtz S, et al: Chemically induced mouse models of intestinal inflammation. Nat Protoc 2007;2:541-546.

20 Perše M, Cerar A: Dextran sodium sulphate colitis mouse model: traps and tricks. J Biomed Biotechnol 2012;2012:718617.

21 Strober W, Fuss IJ, Blumberg RS: The immunology of mucosal models of inflammation. Annu Rev Immunol 2002;20:495-549.

22 Neurath MF, Travis SP: Mucosal healing in inflammatory bowel diseases: a systematic review. Gut 2012;61:1619-1635.

23 Scharl M, et al: Protein tyrosine phosphatase $\mathrm{N} 2$ regulates $\mathrm{TNF} \alpha$-induced signalling and cytokine secretion in human intestinal epithelial cells. Gut 2011;60:189-197.

24 Scharl M, Hruz P, McCole DF: Protein tyrosine phosphatase non-receptor type 2 regulates IFN- $\gamma$-induced cytokine signaling in THP-1 monocytes. Inflamm Bowel Dis 2010; 16:2055-2064.
25 Scharl M, Rudenko I, McCole DF: Loss of protein tyrosine phosphatase N2 potentiates epidermal growth factor suppression of intestinal epithelial chloride secretion. Am J Physiol Gastrointest Liver Physiol 2010;299:G935G945.

26 Lemieux E, et al: Constitutive activation of the MEK/ERK pathway inhibits intestinal epithelial cell differentiation. Am J Physiol Gastrointest Liver Physiol 2011;301:G719-G730.

27 Caballero-Franco C, et al: Tuning of protein kinase circuitry by p38a is vital for epithelial tissue homeostasis. J Biol Chem 2013;288: 23788-23797.

28 Spalinger MR, et al: PTPN2 controls differentiation of $\mathrm{CD}^{+} \mathrm{T}$ cells and limits intestinal inflammation and intestinal dysbiosis. Mucosal Immunol 2015;8:918-929.

29 Wiede F, La Gruta NL, Tiganis T: PTPN2 attenuates T-cell lymphopenia-induced proliferation. Nat Commun 2014;5:3073.

30 Wiede F, et al: PTPN2 restrains $\mathrm{CD}^{+} \mathrm{T}$ cell responses after antigen cross-presentation for the maintenance of peripheral tolerance in mice. J Autoimmun 2014;53:105-114.

31 Cool DE, et al: cDNA isolated from a human T-cell library encodes a member of the protein-tyrosine-phosphatase family. Proc Natl Acad Sci U S A 1989;86:5257-5261. 